\title{
Los(as) estudiantes de Psicología y su relación con la Metodología de la Investigación
}

\author{
Psychology students and their relationship with Research Methodology
}

Rosa M. Huerta-Mata ${ }^{a}$

\begin{abstract}
:
In March 2019, a qualitative instrument was applied to 46 students of the 4th semester of the Bachelor's Degree in Psychology of the Actopan Higher School, of the Autonomous University of the State of Hidalgo. The exercise consisted of students writing a letter to themselves expressing their experiences in the Psychology career, it was suggested that they expose what disappoints them and / or bothers them of study the Bachelor's degree.

The present qualitative work aims to analyze the ambiguity (like / dislike) reported in the letters elaborated by the participants on the subjects concerning the research methodology, and how this can be related to their school trajectory. It is proposed to carry out a broader analysis of the challenges faced by students in taking the subjects of the field of methodology, to prevent and / or correct that research becomes a "bottleneck" for the development of the students of the Bachelor of Psychology.
\end{abstract}

Keywords: Research Methodology, university students, Degree in Psychology

\begin{abstract}
Resumen:
En marzo de 2019, se aplicó un instrumento cualitativo a 46 alumnos(as) de $4^{\circ}$ semestre de la Licenciatura en Psicología de la Escuela Superior de Actopan, de la Universidad Autónoma del Estado de Hidalgo. El ejercicio consistió en que los(as) estudiantes escribieran una carta a sí mismos(as) donde expresaran sus experiencias en la carrera de Psicología, en uno de los apartados del documento se sugirió que exteriorizaran lo que les decepciona y/o molesta de estudiar la Licenciatura.

El presente trabajo de corte cualitativo pretende analizar la ambigüedad (agrado/desagrado), que reportan en las cartas que laboraron los(as) participantes, sobre las materias concernientes a la metodología de investigación, y la relación que ello puede tener con su trayectoria escolar.

Se propone realizar un análisis más amplio sobre los retos que enfrentan las y los estudiantes al cursar las materias del ámbito de metodología de la investigación, para prevenir y/o corregir que la investigación se convierta en "cuello de botella" para el desarrollo de los(as) alumnos(as) de la Licenciatura en Psicología.
\end{abstract}

\section{Palabras Clave:}

Metodología de la Investigación, universitarios(as), Licenciatura en Psicología

\section{Introducción}

Analizar las trayectorias escolares de los(as) estudiantes de educación superior permite identificar sus circunstancias académicas, lo cual, se puede llevar a cabo en tres momentos: al ingreso, en el transcurso y al egreso de la institución. En la etapa de tránsito es posible medir el rezago y la deserción escolar e identificar situaciones de riesgo, así como, los elementos que permiten el logro de una permanencia regular 0 adecuada (Casillas y Badillo, 2015).

Las investigaciones sobre la trayectoria escolar han explorado a través de la metodología cuantitativa el desempeño académico de los(as) estudiantes por medio de la obtención de índices de aprobación y reprobación (Chain Revuelta y Ramírez, 1997; López y Ávila, 2015). Sin embargo, es indispensable indagar la trayectoria académica más allá de las calificaciones y su relación con la eficiencia terminal, es primordial atender a las

\footnotetext{
a Autor de Correspondencia, Universidad Autónoma del Estado de Hidalgo, Escuela Superior de Actopan, ORCID: 0000-0003-4661-5855 
características de diversidad de los(as) universitarios(as), a partir de darles voz a sus experiencias en este sistema escolar.

El programa de la Licenciatura en Psicología de la Escuela Superior de Actopan es de tipo científicopráctico, lo que corresponde a una de las clasificaciones de la Asociación Nacional de Instituciones de Educación Superior (ANUIES). Por tanto, uno de los objetivos que se procura alcanzar en la Licenciatura es el que los(as) estudiantes logren "comunicar el conocimiento de la ciencia psicológica, mediante la elaboración de proyectos, para socializar en foros, simposios y coloquios, los descubrimientos de la investigación científica" (Universidad Autónoma del Estado de Hidalgo. Licenciatura en Psicología. Plan de clases 2015. Objetivos curriculares, 2019). De modo que, el desarrollo de habilidades, saberes y actitudes para la investigación son elementos del perfil de egreso de la Licenciatura, para ello se imparten asignaturas relacionadas con la investigación, como lo podemos evidenciar en el mapa curricular. (Ver tabla I).

Tabla 1. Materias relacionadas con metodología de la investigación.

\begin{tabular}{|l|l|}
\hline $\begin{array}{l}\text { Fundamentos de la metodología de la } \\
\text { investigación }\end{array}$ & 3er semestre \\
\hline Metodología cualitativa & $4^{\circ}$ semestre \\
\hline Metodología cuantitativa & $4^{\circ}$ semestre \\
\hline Análisis de datos cualitativos & $5^{\circ}$ semestre \\
\hline Estadística aplicada a la psicología & $5^{\circ}$ semestre \\
\hline Seminario de investigación I & $7^{\circ}$ semestre \\
\hline Seminario de investigación II & $8^{\circ}$ semestre \\
\hline
\end{tabular}

Fuente: elaboración propia con datos del plan de clases 2015 (Universidad Autónoma del Estado de Hidalgo. Licenciatura en Psicología, 2019).

El presente trabajo de corte cualitativo pretende analizar la ambigüedad (agrado/desagrado) respecto a las materias relacionadas con la metodología de la investigación, y cómo esta situación puede relacionarse con la trayectoria escolar de los(as) estudiantes de cuarto semestre de la Licenciatura en Psicología de la Escuela Superior de Actopan.

\section{Metodología}

En marzo de 2019, se aplicó un instrumento cualitativo a 46 alumnos(as) de $4^{\circ}$ semestre $(9$ hombres y 37 mujeres), pertenecientes a los turnos matutino y vespertino de la Licenciatura en Psicología de la Escuela Superior de Actopan, la cual, pertenece a la Universidad Autónoma del Estado de Hidalgo y se encuentra ubicada en la región del Valle del Mezquital.
Los(as) universitarios(as) participaron de manera voluntaria y firmaron un consentimiento informado. El ejercicio consistió en que los(as) estudiantes escribieran una carta a sí mismos(as) donde expresaran sus experiencias en la carrera de Psicología, se sugirió que en uno de los apartados de su documento expusieran lo que les decepciona $\mathrm{y} / \mathrm{o}$ molesta de estudiar la Licenciatura.

\section{Resultados}

Los contenidos curriculares relacionados con la metodología de la investigación son un factor recurrente de molestia y decepción al estudiar la carrera de Psicología, así mismo, son varias las razones por las que los(as) participantes manifiestan estos sentimientos.

Lo que más me decepciona de la carrera es la investigación, ya que es una cuestión que me genera estrés y que no entiendo muy bien, aunque sé que es una rama dentro de la Psicología y que es una base para desarrollar más aspectos dentro de la misma, es un método que no me gusta tanto seguir... (Mujer, estudiante de $4^{0}$ semestre de la Licenciatura en Psicología).

El testimonio anterior coincide con lo que encontraron Ramos-Rodríguez y Sotomayor (2008), respecto a que los(as) estudiantes universitarios(as) en un $60,6 \%$ no elaboran trabajos de investigación, debido a que se auto perciben con deficiencias metodológicas. Del mismo modo, Trejo (2014) identifica que los(as) alumnos(as) de nivel superior muestran dificultad para comprender la teoría que se revisa en las asignaturas relacionadas con metodología de la investigación.

Varios(as) de los participantes en este estudio, señalan que les resulta complejo la aplicación del método científico, aunque explican que comprenden que es importante para su formación profesional. Cabe aclarar que los(as) jóvenes que asisten a la Escuela Superior de Actopan provienen de diversas comunidades rurales y semirrurales pertenecientes al Valle del Mezquital, en Hidalgo, por lo que es una población que puede presentar deprivación sociocultural, lo cual, los(as) pone en desventaja frente a los recursos educativos con los que cuentan otros(as) estudiantes, así pues, las materias que conciernen a la investigación es probable que influyan en marcar jerarquías intelectuales entre los(as) alumnos(as), lo que puede reforzar el desagrado por sus contenidos académicos.

Además, el que los(as) estudiantes configuren un sentimiento de decepción al cursar metodología de la investigación, convierte en prioritario el indagar la relación que los(as) docentes establecen con esta disciplina, así como, con los procesos de motivación que 
dirigen para promover en la población estudiantil el desarrollo de habilidades, actitudes y conocimientos teóricos y prácticos en este ámbito.

Asimismo, es importante que desde el ingreso a la Licenciatura, las estrategias que se aplican con el objetivo de homologar el nivel académico de los(as) nuevos(as) estudiantes, se centren en la aplicación de métodos de estudio, habilidades críticas y de argumentación oral y escrita.

Por otro lado, al estar en la etapa intermedia de la trayectoria escolar, es fundamental crear en los(as) universitarios(as) el agrado por la investigación, sobre todo porque varios(as) expresan el interés por estudiar un posgrado. Así pues, el sentimiento de decepción que surge ante las aproximaciones a la investigación puede dificultar su desempeño en la Licenciatura, lo que tal vez repercuta en sus posibilidades para acceder a grados subsecuentes de especialización.

...no me agrada una de las materias "metodología", esa materia siempre se me ha complicado y no sé cómo podré pasar y seguir adelante, es una materia que para mí considero que es muy difícil, pero aunque sea difícil de verdad espero poder superarla para poder seguir adelante [...] lo que igualmente se me dificulta un poco es hablar frente a otros, pero esas son algunas de las actividades que la materia de "metodología" lleva, además de la investigación y es otra de las razones por las cuales no me gusta. (Mujer, estudiante de $4^{\circ}$ semestre de la Licenciatura en Psicología).

Algunos(as) participantes representan el cursar metodología de la investigación como un factor determinante para continuar de manera adecuada su trayectoria escolar. En las asignaturas correspondientes a esta área del conocimiento, los(as) estudiantes deben generar propuestas de investigación, argumentarlas y defenderlas de manera escrita y oral; por lo tanto, el pensamiento crítico se aplica en su máxima expresión, pues el ejercicio de las funciones mentales superiores de análisis y síntesis son permanentes. Asimismo, el trabajo de investigación es susceptible de observaciones, lo que obliga a los(as) jóvenes a ampliar las capacidades de tolerancia a la crítica y de redirección de ideas para mejorar sus proyectos, elementos esenciales para que no abandonen las tareas investigativas.

En cuanto a lo que no me agrada de la licenciatura, ya sabemos que son las materias de Metodología, por todas las investigaciones que tienes que realizar para llegar a una respuesta de cifras de prevalencia o para saber qué es lo que opinan o piensan los demás sobre cierto tema, siento que es una pérdida de tiempo para mí, que no solo tengo que estudiar sino también tengo que estar en casa realizando tareas del hogar, y a veces no me da tiempo de hacer otras tareas...(Mujer, estudiante de $4^{\circ}$ semestre de la Licenciatura en Psicología).
En cuarto semestre se cursan dos materias relacionadas con el proceso de investigación: metodología cualitativa y metodología cuantitativa (ver tabla I), así pues, es el semestre más saturado en torno a estos contenidos. Por otro lado, los roles de género que desempeñan algunas jóvenes en el ámbito privado es un elemento por considerar al proponer el trabajo con metodologías de investigación, debido al tiempo que las tareas reproductivas restan a la realización de sus trabajos escolares, lo que puede pronunciar las desigualdades académicas de las estudiantes.

Algunos(as) jóvenes expresan ambigüedad ante la investigación, les agrada la labor teórica y documental, sin embargo, consideran que el trabajo de campo les exige un esfuerzo amplio, en cuanto a: tiempo, elaboración de instrumentos, aplicación, análisis de datos; y consideran que es en esta parte del proceso donde pierden el gusto por la investigación, pues dicha tarea solo les permite obtener "unos cuantos" resultados. Al respecto, Trejo (2014) advierte que uno de los obstáculos para que los jóvenes universitarios realicen investigación se centra en lo que denomina una cultura de lo fácil, donde predomina una actitud que expresa el "'no se puede', 'qué difícil', 'qué complicado', 'para qué sirve eso"' (Trejo, 2014:8).

En este sentido, es básico el trabajo en torno a las actitudes de los(as) estudiantes sobre la investigación, lo cual es conveniente realizar desde los primeros semestres a través de la implementación de actividades transversales dentro de las materias de carácter institucional, para que accedan a lógicas que les permitan comprender y valorar este proceso. Asimismo, es necesario que su producción científica cobre utilidad al fomentar su divulgación a través de diversos medios y espacios, que coadyuve al logro de los objetivos académicos de un programa científico-práctico, como lo es el de la Licenciatura en Psicología de la Escuela Superior de Actopan.

Cabe señalar que esta es una exploración inicial desde la perspectiva de los estudiantes, lo que plantea como primordial el indagar sobre la participación de los(as) docentes en la configuración de las mencionadas posturas frente a la investigación.

\section{Propuestas}

Al inicio de la trayectoria escolar se requiere contextualizar con mayor profundidad las situaciones socioculturales y los antecedentes académicos de los(as) estudiantes. Y, con base en ello, estimular el desarrollo de los niveles cognoscitivos de: comprensión, análisis, síntesis, sentido crítico y argumentación. 
Realizar un análisis más amplio sobre los retos que enfrentan las y los universitarios (as) al cursar las materias del ámbito de metodología de la investigación, para prevenir y/o corregir que la investigación se convierta en "cuello de botella" para la trayectoria escolar de los(as) alumnos(as) de la Licenciatura en Psicología. Generar conciencia en los(as) jóvenes sobre la importancia que en el corto, mediano y largo plazo tendrá el que desarrollen conocimientos, habilidades y actitudes para la investigación.

Promover el trabajo colaborativo a través de proyectos mixtos que beneficien las evidencias que se pretenden obtener para ambas materias (impartidas en $4^{\circ}$ semestre), así como, el que las investigaciones sean realizadas en pareja, todo ello, para evitar sobresaturar a los(as) alumnos(as) e incidir en el agrado por la investigación.

\section{Referencias}

[1]Casillas, M. A. (2015). ¿Hacia dónde van los estudios sobre trayectorias escolares en la educación superior? Reflexiones finales. En Juan Carlos Ortega; Rocío López y Esmeralda Alarcón (Coord.). Trayectorias escolares en educación superior. Propuesta metodológica y experiencias en México. Recuperado el 20 de marzo de 2019, de https://www.uv.mx/bdie/files/2016/01/Libro-Trayectorias-escolareseducacion-superior.pdf

[2]Chain Revuelta, R. y Ramírez, C. (1997). Trayectoria escolar: Un estudio sobre la eficiencia terminal en la UV. Revista de la Educación Superior, 26 (102). Recuperado el 20 de marzo de 2019, de http://publicaciones.anuies.mx/revista/102/2/2/es/trayectoria-escolar-unestudio-sobre-la-eficiencia-terminal-en-la-uv

[3] López, Rocío y Ávila, Nancy (2015). Perfil de ingreso y seguimiento académico de una generación universitaria. En Juan Carlos Ortega; Rocío López y Esmeralda Alarcón (Coord.). Trayectorias escolares en educación superior. Propuesta metodológica y experiencias en México. Recuperado el 20 de marzo de 2019, de https://www.uv.mx/bdie/files/2016/01/LibroTrayectorias-escolares-educacion-superior.pdf

[4] Ramos-Rodríguez, Mariana y Sotomayor, Renzo (2008). Realizar o no una tesis: razones de estudiantes de medicina de una universidad pública y factores asociados. Recuperado el 1 de agosto de 2019, de http://www.redalyc.org/articulo.oa?id=36311611013

[5] Trejo, H. (2014). El desafío de jóvenes investigadores ¡Rompiendo obstáculos! Congreso Iberoamericano de Ciencia, Tecnología, Innovación y Educación. Recuperado el 1 de agosto de 2019, de https://www.oei.es/historico/congreso2014/memoriactei/210.pdf

[6] Universidad Autónoma del Estado de Hidalgo. Licenciatura en Psicología (2019). Plan de clases 2015. Recuperado el 20 de marzo de 2019 ,

https://www.uaeh.edu.mx/campus/icsa/oferta/licenciaturas/psicologia/pla n_estudios.html

[7] Universidad Autónoma del Estado de Hidalgo. Licenciatura en Psicología (2019). Plan de clases 2015. Objetivos curriculares. Recuperado el 20 de marzo de 2019, de https://www.uaeh.edu.mx/campus/icsa/oferta/licenciaturas/psicologia/mis ion_vision.html 